Artículo

\title{
InfoStat, InfoGen y SAS para contrastes mutuamente ortogonales en experimentos en bloques completos al azar en parcelas subdivididas
}

\author{
Andrés González Huerta ${ }^{\S}$ \\ Delfina de Jesús Pérez López \\ Martín Rubí Arriaga \\ Francisco Gutiérrez Rodríguez \\ J. Ramón Pascual Franco Martínez \\ Araceli Padilla Lara \\ Facultad de Ciencias Agrícolas-Centro de Investigación y Estudios Avanzados en Fitomejoramiento- \\ Universidad Autónoma del Estado de México. El Cerrillo Piedras Blancas, Estado de México. AP. 435. Tel. \\ y Fax. 722 2965518, ext. 148 (djperezl@uaemex.mx; m_rubi65@yahoo.com.mx; fgrfca@hotmail.com; \\ jrfrancom@uaemex.mx; padillalaraaraceli@hotmail.com). \\ ${ }^{\S}$ Autor para correspondencia: agonzalezh@uaemex.mx.
}

\section{Resumen}

Las series de experimentos (SE) en tiempo y espacio en arreglos de parcelas divididas han sido utilizadas cotidianamente en diversas disciplinas de la ciencia y la tecnología, pero para parcelas subdivididas (PS) existe poca información. Con contrastes mutuamente ortogonales (CMO's) se divide la variabilidad de medias o totales de tratamientos en grupos, dentro de un análisis de varianza en experimentos de uno o más factores. En este estudio se presentan los programas para analizar datos del peso de vaina en verde registrado en habas en una SE en bloques completos al azar en arreglo de PS para correrse en el Sistema para Análisis Estadístico, InfoStat o InfoGen, elaborados por el autor para correspondencia. Se indican los procedimientos para determinar cuántos y cuáles son los coeficientes de cada contraste y como calcular algunos CMO's. Se presenta un análisis de varianza combinado y los CMO's para factores principales y para sus interacciones, pero los tres paquetes estadísticos pueden generar los análisis para cada ensayo. Con la plataforma que se ha elaborado en el presente estudio será más fácil obtener la descomposición de los efectos de factores principales y de sus interacciones en una SE en arreglo de PS con base en la construcción de un conjunto apropiado de polinomios ortogonales o de una combinación entre éstos y los CMO's.

Palabras clave: Vicia faba L., experimentos factoriales, modelo de efectos fijos, Valles Altos del centro de México.

Recibido: julio de 2019

Aceptado: septiembre de 2019 


\section{Introducción}

Cuando se diseñan y analizan experimentos, el análisis de varianza (Anava) se ha utilizado convencionalmente para minimizar el error experimental y estimar confiablemente los efectos entre tratamientos (Juárez y Corona, 1990; Sahagún y Frey, 1990; Sahagún, 1997; Meneses et al., 2004). Las suposiciones que deben satisfacerse es que el modelo lineal elegido describa adecuadamente las observaciones, y que los errores siguen una distribución normal e independiente, con media cero y varianza constante, aunque desconocida (Sahagún, 1990; Sahagún et al., 2008).

El análisis de variables cuantitativas en modelos de efectos fijos, aleatorios o mixtos puede referirse a casos balanceados o desbalanceados (Matzinger et al., 1959; Sahagún, 1998; Montgomery, 2010) con factores cualitativos o cuantitativos como fertilización, densidad de siembra o población, insecticidas, fungicidas, hormonas vegetales, cultivares, localidades, años o sus combinaciones, entre otros (Sahagún y Frey, 1990; Sahagún, 1997; Meneses et al., 2004; González et al., 2007).

Los experimentos factoriales permiten ahorrar recursos, incrementar la precisión de las estimaciones de medias de efectos y hacer posible el estudio de sus interacciones (Sahagún et al., 2008). El empleo de planes de cruzamiento y de diseños experimentales es un procedimiento común en el mejoramiento genético vegetal y animal (Matzinger et al., 1959; Sahagún, 1997), así como en la producción de semillas, la generación, aplicación, transferencia y validación de tecnología (Sahagún y Frey, 1990; Meneses et al., 2004; González et al., 2007; Torres et al., 2017).

El Anava es un prerrequisito en la comparación de medias de tratamiento, pero sus efectos también pueden dividirse en contrastes mutuamente ortogonales (CMO's) cuando se elige uno de los cuatro diseños experimentales básicos o cuando se aplican las series de experimentos en tiempo y espacio con arreglos en franjas y parcelas divididas (Gomez y Gomez, 1984; Martínez, 1988; Sahagún, 1998; Rebolledo, 2002). La formación de CMO’s tiene estas ventajas: a) cada prueba de hipótesis aporta información nueva, independiente; b) la interpretación de resultados es más sencilla; y c) el número máximo estimable es limitado.

En su construcción la guía básica es su congruencia con los objetivos de la investigación, no importa si son o no mutuamente ortogonales, ni cuantos tratamientos sean evaluados (Sahagún et al., 2008). En factoriales incompletos se han empleado las interacciones de mayor grado como error experimental, aunque no es común experimentar con más de cuatro factores y no es frecuente que todas sus interacciones sean significativas (Sahagún et al., 2008; Montgomery, 2010; Walpole et al., 2012). La interacción genotipo x ambiente, los polinomios ortogonales, las diferentes técnicas de regresión y los diversos métodos multivariados también usan el ANAVA (Gomez y Gomez, 1984; Sahagún, 1990; Sahagún et al., 2008; Torres et al., 2017).

La SE en bloques completos al azar (DBCA) en parcelas divididas está descrito en muchas publicaciones, como en Martínez (1988) y Rebolledo (2002), quienes además elaboraron varios programas para el SAS para generar el Anava, la comparación de medias de tratamientos con la prueba de Tukey y los CMO's. El modelo lineal de una SE en DBCA en arreglo de PS fue descrito por Herrera (2011) y por Padilla et al. (2019) pero aún existe poca información para modelos aleatorios y mixtos y particularmente, cuando en parcela grande, mediana o chica se alojan dos factores (Villa et al., 2010). 
El análisis de una SE en parcelas subdivididas está sujeto a más errores cuando se han registrado muchas variables, pero la elaboración de programas para InfoStat, InfoGen y SAS, entre otros, ahorrará tiempo y esfuerzos si el modelo lineal elegido es correcto (Sahagún, 1998; Herrera, 2011; Padilla et al., 2019). El objetivo del presente estudio fue presentar programas para analizar una SE en PS en DBCA con CMO's empleando tres paquetes estadísticos de uso común.

\section{Marco de referencia}

\section{Modelo lineal}

Se establece que: $i=1,2,3, \ldots$, e experimentos; $j=1,2,3, \ldots, r$ repeticiones; $k=1,2,3, \ldots$ a niveles en parcela grande; $1=1,2,3, \ldots$, b niveles en parcela mediana; $m=1,2,3, \ldots, c$ niveles en parcela chica. Así:

$$
\begin{aligned}
\mathrm{Y}_{\mathrm{ijklm}}= & \mu+\alpha_{\mathrm{i}}+\beta_{\mathrm{j}(\mathrm{i})}+\gamma_{\mathrm{k}}+(\alpha \gamma)_{\mathrm{ik}}+\varepsilon_{\mathrm{ijk}}+\delta_{1}+(\gamma \delta)_{\mathrm{kl}}+(\alpha \delta)_{\mathrm{il}}+(\alpha \gamma \delta)_{\mathrm{ikl}}+\varepsilon_{\mathrm{ijkl}}+\theta_{\mathrm{m}}+(\gamma \theta)_{\mathrm{km}}+(\delta \theta)_{\mathrm{lm}}+ \\
& (\gamma \delta \theta)_{\mathrm{klm}}+(\alpha \theta)_{\mathrm{im}}+(\alpha \gamma \theta)_{\mathrm{ikm}}+(\alpha \delta \theta)_{\mathrm{ilm}}+\varepsilon_{\mathrm{ijklm}}
\end{aligned}
$$

Donde: $\mu$ es la gran media aritmética; $\alpha_{\mathrm{i}}$ es el i-ésimo experimento; $\beta_{\mathrm{j}(\mathrm{i})}$ es la j-ésima repetición anidada en el i-ésimo experimento; $\gamma_{\mathrm{k}}$ es la k-ésima fertilización; $\delta_{1}$ es l-ésima densidad de población; $\theta_{\mathrm{m}}$ es el m-ésimo cultivar; $\varepsilon_{\mathrm{ijk}}, \varepsilon_{\mathrm{ijkl}} \mathrm{y} \varepsilon_{\mathrm{ijklm}}$ son los errores a, b y c de parcela grande, mediana y chica, las diez componentes restantes son interacciones viables (Herrera, 2011; Padilla et al., 2019).

\section{Obtención de los coeficientes de los CMO}

Gomez y Gomez (1984); Sahagún et al. (2008) definen un contraste ortogonal como una combinación lineal de efectos de tratamientos. $\mathrm{Si} \mathrm{T}_{1}, \mathrm{~T}_{2}, \mathrm{~T}_{3}, \mathrm{Tt}$ son parámetros desconocidos relacionados con los efectos de $\mathrm{t}$ tratamientos y $\mathrm{C}_{1}, \mathrm{C}_{2}, \mathrm{C}_{3}, \mathrm{C}_{\mathrm{t}}$ son constantes conocidas, denominadas coeficientes de los contrastes, en experimentos monofactoriales, cada contraste $\left(\mathrm{L}_{\mathrm{i}}\right)$ se calcula como:

$\mathrm{L}_{\mathrm{i}}=\mathrm{C}_{1} \mathrm{~T}_{1}+\mathrm{C}_{2} \mathrm{~T}_{2}+\mathrm{C}_{3} \mathrm{~T}_{3}+, \ldots, \mathrm{C}_{\mathrm{t}} \mathrm{T}_{\mathrm{t}}=\sum_{\mathrm{i}=1}^{\mathrm{t}} \mathrm{CiTi}$ con la restricción $\sum_{\mathrm{i}=1}^{\mathrm{t}} \mathrm{Ci}=0$

La suma de cuadrados (SC) de $\mathrm{L}_{\mathrm{i}}$ con un grado de libertad (gl) se calcula como:

$\mathrm{SCL}_{\mathrm{i}}=\frac{\left[\sum_{\mathrm{i}=1}^{\mathrm{t}} \mathrm{CiTi}\right]^{2}}{\mathrm{r} \sum_{\mathrm{i}=1}^{\mathrm{t}} \mathrm{C}_{\mathrm{i}}^{2}}$

Con t tratamientos hay t-1 contrastes ortogonales. Para probar su significancia estadística en un modelo de efectos fijos se compara el cociente que resulta de dividir $\mathrm{SCL}_{\mathrm{i}}$ entre $\mathrm{SC}$ del error. En la prueba de F se utiliza (1, grados de libertad del error), al nivel de significancia elegido ( $p=0.05$ ó $p=0.01)$.

Dos contrastes, $\mathrm{L}_{1}$ y $\mathrm{L}_{2}$, con un grado de libertad (GL), son mutuamente ortogonales si la suma de los productos cruzados de sus coeficientes es igual a cero; es decir: 
$\mathrm{L}_{1}=\mathrm{C}_{11} \mathrm{~T}_{1}+\mathrm{C}_{12} \mathrm{~T}_{2}+\mathrm{C}_{13} \mathrm{~T}_{3}+, \ldots,+\mathrm{C}_{1 \mathrm{t}} \mathrm{T}_{\mathrm{t}}$

$\mathrm{L}_{2}=\mathrm{C}_{21} \mathrm{~T}_{1}+\mathrm{C}_{22} \mathrm{~T}_{2}+\mathrm{C}_{23} \mathrm{~T}_{3}+, \ldots,+\mathrm{C}_{2 \mathrm{t}} \mathrm{T}_{\mathrm{t}}$

$\sum_{i=1}^{t} \mathrm{C} 1 \mathrm{iC} 2 \mathrm{i}=\mathrm{C} 11 \mathrm{C} 21+\mathrm{C} 12 \mathrm{C} 22+\mathrm{C} 13 \mathrm{C} 23+, \ldots,+\mathrm{C} 1 \mathrm{tC} 2 \mathrm{t}=0$

p contrastes con un GL ( $p>2)$ son mutuamente ortogonales si cada par y todos los pares en el grupo son ortogonales. Como el número máximo de contrastes mutuamente ortogonales con un GL es igual al número de GL de tratamientos entonces:

$\mathrm{SCL}_{1}+\mathrm{SCL}_{2}+\mathrm{SCL}_{3}+,+\mathrm{SCL}_{\mathrm{t}-1}=\mathrm{SC}$ de tratamientos

Para calcular los coeficientes de los contrastes mutuamente ortogonales (CMO) en las interacciones de cualquier orden en experimentos factoriales se procede así: 1. Para determinar cuántos coeficientes habrá se multiplica el número de CMO de cada factor; 2. Los coeficientes para cada interacción se obtienen como el producto de sus valores y signos; y 3. Se capturan todos los coeficientes de cada contraste en el programa editor del SAS o en el cuadro de diálogo del InfoStat o del InfoGen.4. Se corre el programa, se verifican las salidas, se realiza un resguardo o se imprimen las salidas.

Con 5 y 2 CMO para los factores A y B habrá 10 posibilidades. Como hay 6 ó 3 coeficientes en los factores A o B, cualquier CMO tendrá 18 coeficientes. Si los coeficientes del primer contraste para

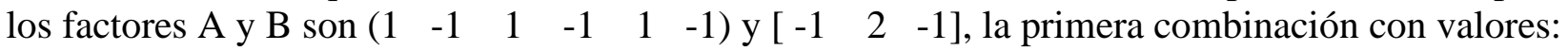

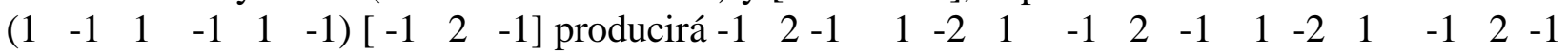
$1-21$.

Nota: se multiplica 1(-1), 1(2), 1(-1), -1(-1), -1(2), -1(-1), .., -1(-1), -1(2), -1(-1).

Para AxC habrá también 10 casos, con 18 coeficientes cada uno; sus valores y signos son iguales a los de A1B1. Así: $\left(\begin{array}{llllll}1 & -1 & 1 & -1 & 1 & -1\end{array}\right)\left[\begin{array}{lll}-1 & 2 & -1\end{array}\right]=\begin{array}{lllllllllllllll}-1 & 2 & -1 & 1 & -2 & 1 & -1 & 2 & -1 & 1 & -2 & 1 & -1 & 2 & -1\end{array}$ $1-21$.

Para la interacción BxC deben obtenerse los 3x3=9 coeficientes para cada uno de los cuatro CMO.

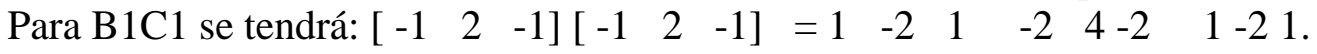

Nota: cada valor se obtiene cómo -1(-1), -1(2), -1(-1), 2(-1), 2(2), 2(-1), -1(-1), -1(2), -1(-1).

En $\mathrm{AxBxC}$, hay $6 \times 3 \times 3=54$ coeficientes para cada uno de los $5 \times 2 \times 2=20$ contrastes mutuamente ortogonales. Para SAS sus valores y signos se obtienen al multiplicar los coeficientes de cada interacción $\mathrm{AxB}$ con los de $\mathrm{C}$, pero para InfoStat estos se generan al multiplicar los valores de $\mathrm{C}$ por los de AxB. Para SAS:

En A1B1C1 sus valores son el producto de $\left(\begin{array}{lllllllllllllll}-1 & 2 & -1 & 1 & -2 & 1 & -1 & 2 & -1 & 1 & -2 & 1 & -1 & 2 & -1\end{array}\right.$

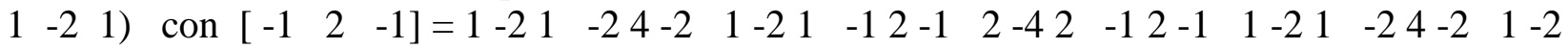

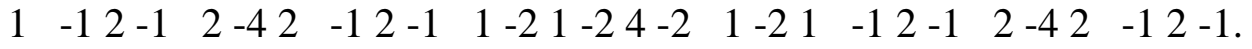




\section{Análisis estadístico}

Los pesos de vaina en verde fueron sometidos a un análisis de varianza combinado. Los procedimientos algebraicos están descritos en Herrera (2011); Padilla et al. (2019). Las salidas se obtuvieron con las versiones descritas en SAS Institute (1989); InfoStat (Balzarini et al., 2008; Di Rienzo et al., 2008) e InfoGen (Balzarini y Di Rienzo, 2016). Adicionalmente, se hizo una subdivisión de los tres efectos principales y de sus cuatro interacciones con contrastes mutuamente ortogonales (Gomez y Gomez, 1984).

\section{Cálculo de algunos CMO}

En Padilla et al. (2019) se muestran los datos del rendimiento en vaina verde utilizados para realizar los siguientes cálculos. Con los totales de $\mathrm{AxB}, \mathrm{AxC}$ y $\mathrm{AxBxC}$ se pueden calcular los $\mathrm{CMO}$ para los tres factores y para la interacción $\mathrm{BxC}$.

Contraste 1 del factor A (FER)

$\mathrm{SC} \mathrm{L1}=\frac{\left(\sum_{i=1}^{c} C i T i\right)^{2}}{e b c r \sum_{i=1}^{c} C i^{2}}=\frac{(365.11-375.06+463.38-344.10+410.86-353.36)^{2}}{2(3)(3)(3)(6)}=\frac{(1239.45-1072.52)^{2}}{324}=86$

Contraste 1 del factor B (DEN)

$\mathrm{SCL} 1=\frac{\left(\sum_{i=1}^{c} C i T i\right)^{2}}{e \operatorname{eacr} \sum_{i=1}^{c} C i^{2}}=\frac{\left[(496.23-2(783.38)+1032.39]^{2}\right.}{2(6)(3)(3)(6)}=\frac{(1528.62-1566.76)^{2}}{648}=2.245$

Contraste 1 del factor C (CUL)

$\mathrm{SCL} 1=\frac{\left(\sum_{i=1}^{c} C i T i\right)^{2}}{e a b r \sum_{i=1}^{c} C i^{2}}=\frac{\left[(819.77-2(741.83)+750.37]^{2}\right.}{2(6)(3)(3)(6)}=\frac{(1570.14-1483.66)^{2}}{648}=11.54$

Contraste 1 de la interacción FER x DEN (AxB)

SCL1 $=\frac{\left(\sum_{i=1}^{c} C i T i\right)^{2}}{e c r \sum_{i=1}^{c} C i^{2}}$, donde: e, c y r son localidades, cultivares y repeticiones, respectivamente.

$=[81.81-70.94+108.36-79.72+82.8-72.57-2(128.61)+2(137.4)-2(147.09)+2(113.16)$

$-2(143.56)+2(113.56)+154.69-116.72+208.03-151.22+184.5-167.23] 2 /[2(3)(3)(36)]$

$=(1548.43-1546.92) 2 / 648=0.0035$.

Contraste 1 de la interacción FER x CUL (AxC)

SCL1 $=\frac{\left(\sum_{i=1}^{c} C \boldsymbol{C} T \boldsymbol{T}\right)^{2}}{e \boldsymbol{e b r} \sum_{i=1}^{c} C \boldsymbol{i}^{2}}$, donde: b son los niveles de la densidad de población.

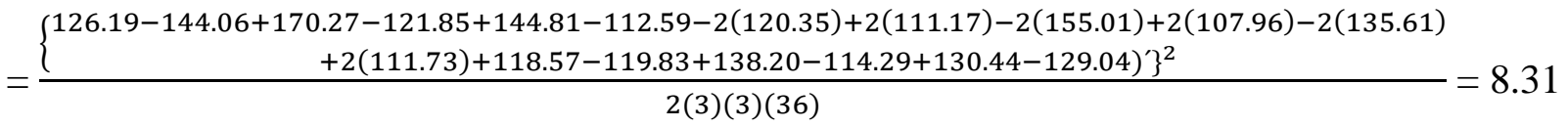


Contraste 1 de la interacción DEN x CUL (B x C).

SCL1 $=\frac{\left(\sum_{i=1}^{c} C i T i\right)^{2}}{e a r \sum_{i=1}^{c} C i^{2}}$, donde: a son los niveles de la fertilización.

$=\frac{[188.54-2(269,27)+361.96-2(152.38)+4(260.93)-2(328.52)+155.28-2(253.18)+341.91]^{2}}{2(6)(3)(36}=5.53$.

Contraste 1 de la interacción FER x DEN x CUL (AxBxC).

$$
\begin{aligned}
& \operatorname{SCL1}=\frac{\left(\sum_{i=1}^{c} C i T i\right)^{2}}{\operatorname{er} \sum_{i=1}^{c} C i^{2}} \\
& {[30.31-2(41.9)+53.98-29.29+2(52.3)-62.47+47.78-2(51.3)+71.19-30.49+2(37.81)-53.55} \\
& +28.84-2(50.23)+65.74-21.83+2(35.73)-55.03-2(26.82)+4(45.81)-2(47.72)+2(15.05)-4(43.92)+2(52.2) \\
& -2(31.55)+4(53.34)-2(70.12)+2(22.7)-4(37.81)+2(47.45)-2(29.74)+4(42.67)-2(63.15)+2(26.47)-4(37.38) \\
& +2(47.88)+24.68-2(40.90)+52.99-26.6+2(41.18)-51.95+29.03-2(42.45)+66.72-26.53+2(37.54)-50.22 \\
& =\frac{+24.17-2(50.66)+55.61-24.27+2(40.45)-65.32]^{2}}{2(3)(216)} \\
& =0.91
\end{aligned}
$$

Los cálculos anteriores y el resto de los CMO que son posibles de estimar se pueden obtener con la siguiente rutina:

\section{Programas para InfoStat e InfoGen}

\section{Etapa 1}

Los datos se ordenan como experimentos (EXP), repeticiones (REP), fertilización (FER), densidad de población (DEN), cultivares (CUL) y variables (s).

\section{Etapa 2}

En menú principal elegir estadísticaslanálisis de varianza. En el cuadro de diálogo definir: Variables dependientes: RVV. Variables de clasificación: EXP REP FER DEN CUL, elegir aceptar.

\section{Etapa 3}

En especificaciones de los términos del modelo escribir:

EXP $\backslash E X P>R E P * F E R ; E X P>R E P \backslash E X P>R E P * F E R ; F E R \backslash E X P>R E P * F E R ;$

EXP*FER $\backslash$ EXP $>$ REP*FER;

EXP $>$ REP*FER;DENIEXP $>$ FER $>$ REP*DEN;DEN*EXP ${ }^{*}$ EXP $>$ FER $>$ REP*DEN;DEN*FER ${ }^{*}$ EX $\mathrm{P}>\mathrm{FER}>\mathrm{REP} * \mathrm{DEN}$;DEN*EXP*FER $\backslash$ EXP $>$ FER $>$ REP*DEN;EXP $>$ FER $>$ REP*DEN;CUL;CUL* EXP;CUL*FER;

CUL*DEN;CUL*EXP*FER;CUL*EXP*DEN;CUL*FER*DEN; elegir aceptar. 
Nota: en el cuadro de diálogo las instrucciones anteriores deben escribirse por separado en cada línea y sin el punto y coma.

\section{Etapa 4}

En el cuadro de diálogo que muestra Análisis de varianza elegir: comparaciones\ contrastes\ tratamientoslescoger efectos\matriz de contrasteslaceptar.

Antes de dar clic en aceptar debe elegirse controlar ortogonalidad. Después de definir si será estimado un efecto principal o una interacción introducir los coeficientes en la matriz de contrastes.

\section{Programa para SAS}

En la base de datos Exp= localidades, A, B y C son fertilización, densidad de población y cultivares y las variables son identificadas como X1, X2, X16.

Data haba; Input Exp rep A B C X1 X2 X3 X4 X5 X6 X7 X8 X9 X10 X11 X12 X13 X14 X15 X16; Cards;

Aquí se capturan los datos en el orden del input.

PROC SORT; BY EXP; PROC GLM; BY EXP; CLASS REP A B C; MODEL X1-X16= REP A REP*A B A*B REP*B(A) C A*C B*C A*B*C; TEST $\mathrm{H}=\mathrm{REP}$ A E=REP*A; TEST $\mathrm{H}=\mathrm{B}$ A*B E$=\mathrm{REP} * \mathrm{~B}(\mathrm{~A})$;

Se genera un análisis de varianza individual con glm y se calculan los contrastes mutuamente ortogonales para efectos principales, se usan los errores de parcela grande y mediana para las pruebas de $\mathrm{f}$.

CONTRAST 'A1, A3,A5 vs A2,A4,A6' A 1 -1 1 -1 1 -1/E=REP*A;

CONTRAST 'A1 vs A3, A5' A $20-10-10 / E=R E P * A$;

CONTRAST 'A3 vs A5' A $0010-10 / \mathrm{E}=\mathrm{REP} * \mathrm{~A}$;

CONTRAST 'A2 vs A4, A6' A $020-10$-1/E= REP *A;

CONTRAST 'A4 vs A6' A $00010-1 / \mathrm{E}=\mathrm{REP} * \mathrm{~A}$;

CONTRAST 'B2 vs B1, B3' B -1 2 -1/E= REP *B(A);

CONTRAST 'B1 vs B3' B $10-1 / \mathrm{E}=\mathrm{REP} * \mathrm{~B}(\mathrm{~A})$;

Los contrastes del factor c se prueban con el residual del modelo, que es el error de parcela chica; no es necesario que se especifiquen al final.

CONTRAST 'C2 vs C1, C3' C -1 2-1;

CONTRAST 'C1 vs C3' C 10 -1;

Los contrastes de la interacción axb se prueban con el error de parcela mediana. 


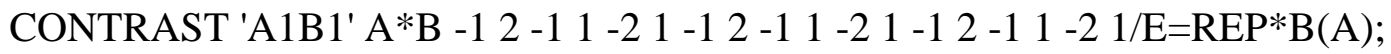

CONTRAST 'A2B1' A*B -2 4 -2 $00001-21000001$-2 1000 0/E=REP*B(A);

CONTRAST 'A3B1' A*B $0000000-12$-1 00001 -2 1000 0/E=REP*B(A);

CONTRAST 'A4B1' A*B $0000-24-200001-2100001$-2 1/E=REP*B(A);

CONTRAST 'A5B1' A*B $00000000000-12-100001$-2 1/E=REP*B(A);

CONTRAST 'A1B2' A*B $10-1$-1 01110 -1 0 -1 01110 -1 -1 0 1/E=REP*B(A);

CONTRAST 'A2B2' A*B $20-2000-1010000-1001000$ 0/E=REP*B(A);

CONTRAST 'A3B2' A*B $0000000010-10000-1001000$ 0/E=REP*B(A);

CONTRAST 'A4B2' A*B $000200-20000-10010000-100$ 1/E=REP*B(A);

CONTRAST 'A5B2' A*B $000000000000100-10000-10$ 1/E=REP*B(A);

Los errores de la interacción axc se prueban con el residual del modelo, por lo que no es necesario que se indiquen al final.

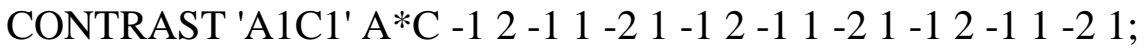

CONTRAST 'A2C1' A*C -2 4 -2 00001 -2 100001 -2 10000 ;

CONTRAST 'A3C1' A*C $0000000-12-100001-21000$;

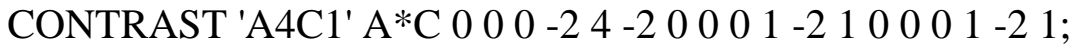

CONTRAST 'A5C1' A*C $000000000000-12-100001-2$;;

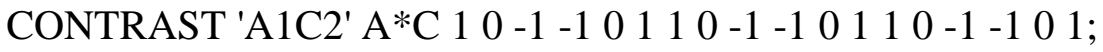

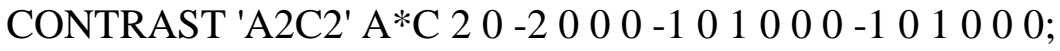

CONTRAST 'A3C2' A*C $0000000010-10000-1001000$;

CONTRAST 'A4C2' A*C $0000220-20000-10010000-1001$;

CONTRAST 'A5C2' A*C $0000000000010-10000-101$;

CONTRAST 'B1C1' B*C 1 -2 1 - -2 - 4 -2 1 - -2 1;

CONTRAST 'BIC2' B*C -1 $01220-2$-1 01 ;

CONTRAST 'B2C1' B*C -1 2 -1 $00001-2$ 1;

CONTRAST 'B2C2' B*C $10-10000-1001$;

Los coeficientes de la interacción axbxc se prueban con el error de parcela chica o residual del modelo y no se indican al final.

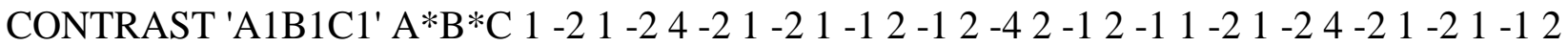

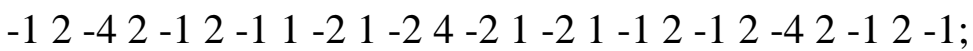

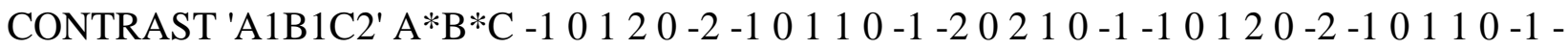
$20210-1-101200-2-101110-1-202210-1$;

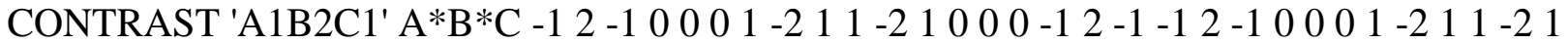

$0000-12-1-12-100001-211-210000-12-1$;

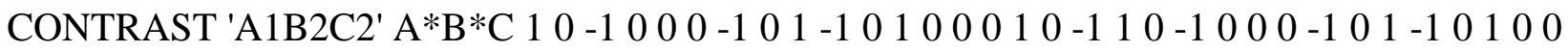
$010-110-10000-1001-1001000010-1$;

CONTRAST 'A2B1C1' A*B*C 2 -4 2 -4 8 -4 2 -4 $2000000000000-12-12-42-12-100000$ $000000-12-12-42-12-10000000000000$;

CONTRAST 'A2B1C2' A*B*C -2 0240 -4 -2 $0200000000000010-1-202210-1000000$ $000010-1-20210-1000000000$;

CONTRAST 'A2B2C1' A*B*C -2 4 -2 $00002-4200000000000001-2100000-12-11000000$ $00001-210000-12-10000000000$; 
CONTRAST 'A2B2C2' A*B*C $20-20000-2020000000000000-1001000010-10000000$ $000-10100010-1000000000$;

CONTRAST 'A3B1C1' A*B*C $0000000000000000000000001-21-24-21-2100000000$ $000-12-12-42-12-100000000000$;

CONTRAST 'A3B1C2' A*B*C $000000000000000000000000-1001220-2-10010000000$ $00010-1-20210-1000000000$;

CONTRAST 'A3B2C1' A*B*C $000000000000000000000000-12-1100001-2100000000$ $0001-210000-12-10000000000$;

CONTRAST 'A3B2C2' A*B*C $000000000000000000000000110-110000-1001000000000$ $00-10100010-1000000000$;

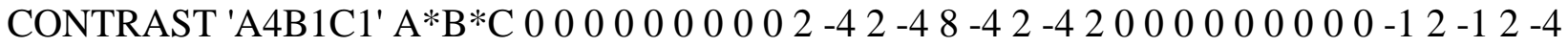
$2-12-100000000000-12-12-42-12-1$;

CONTRAST 'A4B1C2' A*B*C $00000000000-202240-4-200200000000000010-1-202$ $10-10000000000010-1-20210-1$;

CONTRAST 'A4B2C1' A*B*C $000000000000-24-2000002-4200000000000001-210000$ $-12-1000000000001-210000-12-1$;

CONTRAST 'A4B2C2' A*B*C $000000000000200-200000-20020000000000000-10010000$ $10-100000000000-101000010-1$;

CONTRAST 'A5B1C1' A*B*C $00000000000000000000000000000000001-21-24-2$ $1-210000000000-12-12-42-12-1$;

CONTRAST 'A5B1C2' A*B*C $0000000000000000000000000000000000000-101200-2$ $10100000000010-1-20210-1$;

CONTRAST 'A5B2C1' A*B*C $0000000000000000000000000000000000000-12-100001$ $-210000000001-21000-12-1$;

CONTRAST 'A5B2C2' A*B*C $0000000000000000000000000000000000000010-10000-1$ $01000000000-10100010-1$;

PROC GLM; CLASS EXP REP A B C;

MODEL X1-X16= EXP REP(EXP) A A*EXP REP*A(EXP) B A*B B*EXP A*B*EXP REP*B(A EXP) C A*C B*C A*B*C C*EXP A*C*EXP B*C*EXP;

TEST $\mathrm{H}=\mathrm{EXP}$ A REP(EXP) EXP*A E=REP*A(EXP);

TEST $\mathrm{H}=\mathrm{B}$ A*B B*EXP A*B*EXP E=REP*B(A EXP);

Se obtiene un análisis de varianza combinado con partición de efectos principales con contrastes mutuamente ortogonales.

CONTRAST 'A1, A3, A5 vs A2, A4, A6' A 1 -1 1 -1 1 -1/E=Rep*A(Exp);

CONTRAST 'A1 vs A3, A5' A $20-10-1$ 0/E=Rep*A(Exp);

CONTRAST 'A3 vs A5' A 0010 -1 0/E=Rep*A(Exp);

CONTRAST 'A2 vs A4, A6' A 020 -1 0 -1/E=Rep*A(Exp);

CONTRAST 'A4 vs A6' A 000010 -1/E=Rep*A(Exp);

CONTRAST 'B2 vs B1, B3' B -1 2 -1/E=Rep*B(A Exp);

CONTRAST 'B1 vs B3' B $10-1 / \mathrm{E}=\mathrm{Rep} * \mathrm{~B}(\mathrm{~A}$ Exp);

CONTRAST 'C2 vs C1, C3' C -1 $2-1$;

CONTRAST 'C1 vs C3' C 10 -1;

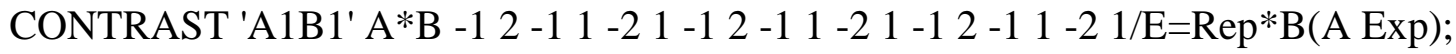


CONTRAST 'A2B1' A*B -2 4 -2 00001 -2 100001 -2 1000 0/E=REP*B(A EXP); CONTRAST 'A3B1' A*B $00000000-12-100001-21000$ 0/E=REP*B(A EXP);

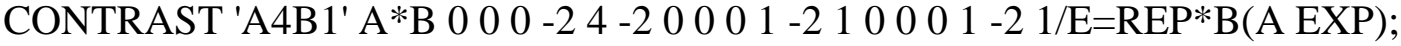
CONTRAST 'A5B1' A*B $000000000000-12$-1 000001 -2 1/E=REP*B(A EXP);

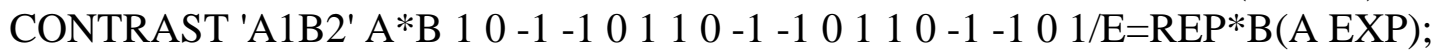
CONTRAST 'A2B2' A*B $20-20000-1001000-1001000$ 0/E=REP*B(A EXP); CONTRAST 'A3B2' A*B $0000000010-10000-1001000$ 0/E=REP*B(A EXP);

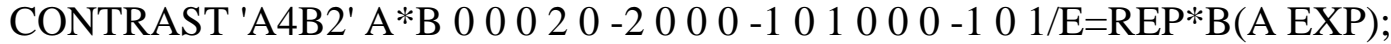
CONTRAST 'A5B2' A*B $000000000000100-10000-10$ 1/E=REP*B(A EXP);

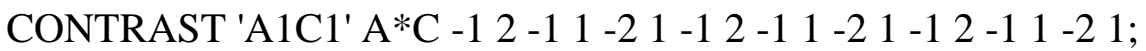
CONTRAST 'A2C1' A*C -2 4 -2 $00001-2100001-21000$; CONTRAST 'A3C1' A*C $0000000-12-100001-21000$;

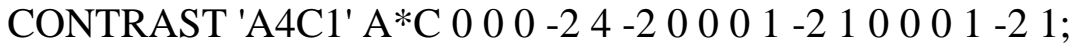
CONTRAST 'A5C1' A*C $00000000000-12-1000001-21$;

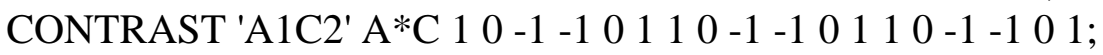
CONTRAST 'A2C2' A*C 20 -2 $0000-1010000-1001000$; CONTRAST 'A3C2' A*C $000000010-10000-1010000$; CONTRAST 'A4C2' A*C $000020-20000-1010000-101$; CONTRAST 'A5C2' A*C $0000000000010-10000-1001$; CONTRAST 'B1C1' B*C 1 -2 1 -2 4 -2 1 -2 1 ; CONTRAST 'BIC2' B*C -1 $01220-2$ - 10 1;

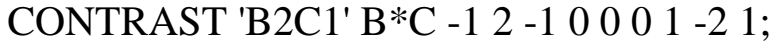
CONTRAST 'B2C2' B*C $10-10000-1001$;

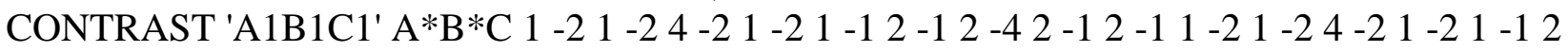

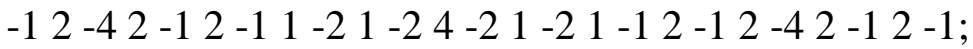

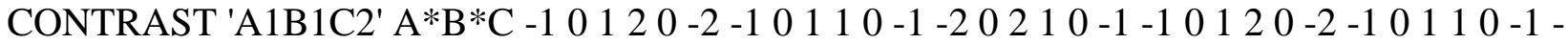
$20210-1-100120-2-100110-1$-2 $02210-1$;

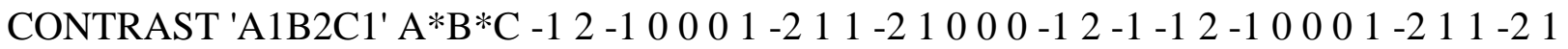
$000-12-1-12-100001-2111-210000-12-1$;

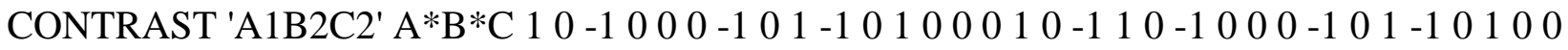
$010-1100-10000-1001-1001000010-1$;

CONTRAST 'A2B1C1' A*B*C $2-42-48$-4 2-4 $2000000000000-12-12-42-12-100000$ $00000-12-12-42-12-1000000000000$;

CONTRAST 'A2B1C2' A*B*C -2 $02440-4-202200000000000010-1-200210-1000000$ $000010-1-20210-1000000000$;

CONTRAST 'A2B2C1' A*B*C -2 4 -2 $00002-4200000000000001-2100000-12-1000000$ $00001-210000-12-10000000000$;

CONTRAST 'A2B2C2' A*B*C $20-20000-2020000000000000-100100001010-10000000$ $000-10100010-1000000000$;

CONTRAST 'A3B1C1' A*B*C $0000000000000000000000001-21-24-21-2100000000$ $000-12-12-42-12-100000000000$;

CONTRAST 'A3B1C2' A*B*C $000000000000000000000000-1001220-2-100100000000$ $00010-1-20210-1000000000$;

CONTRAST 'A3B2C1' A*B*C $0000000000000000000000-12-100001-210000000$ $0001-210000-12-10000000000$;

CONTRAST 'A3B2C2' A*B*C $0000000000000000000000000100-100000-1001000000000$ $00-10100010-1000000000$; 
CONTRAST 'A4B1C1' A*B*C $0000000000002-42-448-42-420000000000000-12-12$-4 $2-12$-1 $00000000000-12-12-42-12-1$;

CONTRAST 'A4B1C2' A*B*C $00000000000-202240-4-2002000000000000100-1-202$ $10-10000000000010-1-20210-1$;

CONTRAST 'A4B2C1' A*B*C $000000000000-24-2000002-4200000000000001-210000$ $-12-10000000000001-210000-12-1$;

CONTRAST 'A4B2C2' A*B*C $00000000000020-200000-2002000000000000-10010000$ $10-100000000000-101000010-1$;

CONTRAST 'A5B1C1' A*B*C $00000000000000000000000000000000000001-21-24-2$ 1 -2 $100000000000-12-12-42-12-1$;

CONTRAST 'A5B1C2' A*B*C $0000000000000000000000000000000000-100120-2$ $10100000000010-1-20210-1$;

CONTRAST 'A5B2C1' A*B*C $00000000000000000000000000000000000000-12-100001$ $-21000000000001-210000-12-1$;

CONTRAST 'A5B2C2' A*B*C $00000000000000000000000000000000000010-1000-1$ $01000000000-10100010-1$;

RUN;

Algunos resultados obtenidos con los tres paquetes estadísticos.

\begin{tabular}{cccccc}
\hline Fuente de & $\begin{array}{c}\text { Grados de } \\
\text { variación }\end{array}$ & $\begin{array}{c}\text { Suma de } \\
\text { cuadrados }\end{array}$ & $\begin{array}{c}\text { Cuadrados } \\
\text { medios }\end{array}$ & $\begin{array}{c}\text { Valor } \\
\text { de F }\end{array}$ & $\begin{array}{c}\text { Valor } \\
\text { de } p\end{array}$ \\
\hline Modelo & 159 & 4571.71 & 28.75 & 6.58 & 0.0001 \\
Experimentos (Exp) & 1 & 37.13 & 37.13 & 3.7 & 0.0686 \\
Rep (Exp) & 4 & 1650.68 & 412.67 & 41.16 & 0.0001 \\
Fertilización (Fer) & 5 & 185.09 & 37.02 & 3.69 & 0.0157 \\
Exp x Fer & 5 & 150.73 & 30.15 & 3.01 & 0.0349 \\
Error a & 20 & 200.5 & 10.02 & 2.29 & \\
Densidad (Den) & 2 & 1333.63 & 666.63 & 61.46 & 0.0001 \\
Fer x Den & 10 & 49.9 & 4.99 & 0.46 & 0.9073 \\
Exp x Den & 2 & 67.69 & 33.84 & 3.12 & 0.0532 \\
Exp x Fer x Den & 10 & 106.91 & 10.69 & 0.99 & 0.4684 \\
Error b & 48 & 520.61 & 10.85 & 2.48 & \\
Cultivares (Cul) & 2 & 33.85 & 16.92 & 3.87 & 0.0228 \\
Cul x Fer & 10 & 50.63 & 5.06 & 1.16 & 0.3228 \\
Cul x Den & 4 & 7.92 & 1.98 & 0.45 & 0.7703 \\
Cul x Fer x Den & 20 & 59.49 & 2.97 & 0.68 & 0.8416 \\
Cul x Exp & 2 & 56.25 & 28.13 & 6.43 & 0.002 \\
Cul x Exp x Fer & 10 & 53.98 & 5.4 & 1.23 & 0.2725 \\
Cul x Exp x Den & 4 & 7.08 & 1.77 & 0.41 & 0.8048 \\
Error c & 164 & 716.96 & 4.37 & & \\
Total & 323 & 5288.67 & & & \\
\hline
\end{tabular}


Contrastes mutuamente ortogonales para fertilización (parcela grande).

\begin{tabular}{cccccl}
\hline Fer (A) & $\begin{array}{c}\text { Grados de } \\
\text { libertad }\end{array}$ & $\begin{array}{c}\text { Suma de } \\
\text { cuadrados }\end{array}$ & $\begin{array}{c}\text { Valor } \\
\text { de F }\end{array}$ & $\begin{array}{c}\text { Valor } \\
\text { de } p\end{array}$ & $\begin{array}{c}\text { Error de parcela } \\
\text { grande }\end{array}$ \\
\hline A1 & 1 & 86.01 & 8.58 & 0.0083 & Exp>Rep>Fer \\
A2 & 1 & 64.11 & 6.39 & 0.02 & Exp>Rep>Fer \\
A3 & 1 & 25.64 & 2.56 & 0.1255 & Exp>Rep>Fer \\
A4 & 1 & 8.56 & 0.85 & 0.3665 & Exp>Rep>Fer \\
A5 & 1 & 0.79 & 0.08 & 0.7813 & Exp>Rep>Fer \\
Total & 5 & 185.1 & 3.69 & 0.0157 & Exp>Rep>Fer \\
\hline
\end{tabular}

Contrastes mutuamente ortogonales para densidad de población (parcela mediana).

\begin{tabular}{cccccc}
\hline Den $(\mathrm{B})$ & $\begin{array}{c}\text { Grados de } \\
\text { libertad }\end{array}$ & $\begin{array}{c}\text { Suma de } \\
\text { cuadrados }\end{array}$ & $\begin{array}{c}\text { Valor } \\
\text { de F }\end{array}$ & $\begin{array}{c}\text { Valor } \\
\text { de } p\end{array}$ & $\begin{array}{c}\text { Error de parcela } \\
\text { mediana }\end{array}$ \\
\hline B1 & 1 & 2.25 & 0.21 & 0.65 & Exp>Fer $>$ Rep*Den \\
B2 & 1 & 1331.02 & 122.72 & 0.001 & $\begin{array}{c}\text { Exp }>\text { Fer }>\text { Rep*Den } \\
\text { Exp }>\text { Fer }>\text { Rep*Den }\end{array}$ \\
Total & 2 & 1333.27 & 61.46 & 0.0001 & . \\
\hline
\end{tabular}

Contrastes mutuamente ortogonales para cultivares de haba (parcela chica).

\begin{tabular}{cccccc}
\hline Cul (C) & $\begin{array}{c}\text { Grados de } \\
\text { libertad }\end{array}$ & $\begin{array}{c}\text { Suma de } \\
\text { Cuadrados }\end{array}$ & $\begin{array}{c}\text { Valor de } \\
\text { F }\end{array}$ & $\begin{array}{c}\text { Valor de } \\
p\end{array}$ & $\begin{array}{c}\text { Error de parcela } \\
\text { chica }\end{array}$ \\
\hline C1 & 1 & 11.54 & 2.64 & 0.1061 & Residual del modelo \\
C2 & 1 & 22.3 & 5.1 & 0.0252 & Residual del modelo \\
Total & 2 & 33.84 & 3.87 & 0.0228 & Residual del modelo \\
\hline
\end{tabular}

Contrastes mutuamente ortogonales para la interacción fertilización x densidad (AxB).

\begin{tabular}{|c|c|c|c|c|c|}
\hline $\begin{array}{c}\text { DenxFer } \\
\text { o AxB }\end{array}$ & $\begin{array}{c}\text { Grados de } \\
\text { libertad }\end{array}$ & $\begin{array}{c}\text { Suma de } \\
\text { Cuadrados }\end{array}$ & $\begin{array}{c}\text { Valor de } \\
\text { F }\end{array}$ & $\begin{array}{c}\text { Valor de } \\
p\end{array}$ & $\begin{array}{c}\text { Error para la } \\
\text { interacción }\end{array}$ \\
\hline $\mathrm{C} 1$ & 1 & 0.003 & 0.0003 & 0.98 & Exp $>$ Fer $>$ Rep*Den \\
\hline $\mathrm{C} 2$ & 1 & 2.96 & 0.27 & 0.6 & Exp $>$ Fer $>$ Rep*Den \\
\hline $\mathrm{C} 3$ & 1 & 8.18 & 0.75 & 0.38 & Exp $>$ Fer $>$ Rep*Den \\
\hline $\mathrm{C} 4$ & 1 & 12.94 & 1.19 & 0.28 & Exp $>$ Fer $>$ Rep*Den \\
\hline $\mathrm{C} 5$ & 1 & 0.3 & 0.03 & 0.86 & Exp $>$ Fer $>$ Rep*Den \\
\hline C6 & 1 & 0.7 & 0.6 & 0.8 & Exp $>$ Fer $>$ Rep*Den \\
\hline $\mathrm{C} 7$ & 1 & 14.32 & 1.32 & 0.25 & Exp $>$ Fer $>$ Rep*Den \\
\hline $\mathrm{C} 8$ & 1 & 0.06 & 0.01 & 0.94 & Exp $>$ Fer $>$ Rep*Den \\
\hline C9 & 1 & 2.99 & 0.28 & 0.6 & Exp $>$ Fer $>$ Rep*Den \\
\hline $\mathrm{C} 10$ & 1 & 7.45 & 0.69 & 0.41 & Exp $>$ Fer $>$ Rep*Den \\
\hline Total & 10 & 49.9 & 0.90 & 0.9 & Exp $>$ Fer $>$ Rep*Den \\
\hline
\end{tabular}


Contrastes mutuamente ortogonales para la interacción fertilización x cultivares o AxC.

\begin{tabular}{cccccl}
\hline $\begin{array}{c}\text { CulxFer } \\
\text { ó AxC }\end{array}$ & $\begin{array}{c}\text { Grados de } \\
\text { libertad }\end{array}$ & $\begin{array}{c}\text { Suma de } \\
\text { cuadrados }\end{array}$ & $\begin{array}{c}\text { Valor de } \\
\text { F }\end{array}$ & $\begin{array}{c}\text { Valor de } \\
p\end{array}$ & Error de la interacción \\
\hline C1 & 1 & 8.31 & 1.9 & 0.16 & Residual del modelo \\
C2 & 1 & 0.05 & 0.01 & 0.91 & Residual del modelo \\
C3 & 1 & 0.14 & 0.03 & 0.85 & Residual del modelo \\
C4 & 1 & 3.08 & 0.71 & 0.4 & Residual del modelo \\
C5 & 1 & 0.02 & 0.004 & 0.94 & Residual del modelo \\
C6 & 1 & 6.94 & 1.59 & 0.2 & Residual del modelo \\
C7 & 1 & 4.51 & 1.03 & 0.31 & Residual del modelo \\
C8 & 1 & 4.35 & 1 & 0.32 & Residual del modelo \\
C9 & 1 & 15.23 & 3.48 & 0.06 & Residual del modelo \\
C10 & 1 & 8.01 & 1.83 & 0.17 & Residual del modelo \\
Total & 10 & 50.64 & 1.16 & 0.32 & Residual del modelo \\
\hline
\end{tabular}

Contrastes mutuamente ortogonales para la interacción densidad x cultivares o BxC.

\begin{tabular}{cccccc}
\hline $\begin{array}{c}\text { CulxDen } \\
\text { o BxC }\end{array}$ & $\begin{array}{c}\text { Grados de } \\
\text { libertad }\end{array}$ & $\begin{array}{c}\text { Suma de } \\
\text { cuadrados }\end{array}$ & $\begin{array}{c}\text { Valor de } \\
\text { F }\end{array}$ & $\begin{array}{c}\text { Valor de } \\
p\end{array}$ & $\begin{array}{c}\text { Error de la } \\
\text { interacción }\end{array}$ \\
\hline C1 & 1 & 5.54 & 1.27 & 0.26 & Residual del modelo \\
C2 & 1 & 0.14 & 0.03 & 0.85 & Residual del modelo \\
C3 & 1 & 1.03 & 0.24 & 0.62 & Residual del modelo \\
C4 & 1 & 1.21 & 0.28 & 0.59 & Residual del modelo \\
Total & 4 & 7.92 & 0.45 & 0.77 & Residual del modelo \\
\hline
\end{tabular}

Contrastes mutuamente ortogonales para la interacción fertilización x densidad x cultivares.

\begin{tabular}{cccccc}
\hline $\begin{array}{c}\text { CulxDenxFer } \\
\text { o AxBxC }\end{array}$ & $\begin{array}{c}\text { Grados de } \\
\text { libertad }\end{array}$ & $\begin{array}{c}\text { Suma de } \\
\text { cuadrados }\end{array}$ & $\begin{array}{c}\text { Valor de } \\
\text { F }\end{array}$ & $\begin{array}{c}\text { Valor de } \\
p\end{array}$ & $\begin{array}{c}\text { Error para la } \\
\text { interacción }\end{array}$ \\
\hline C1 & 1 & 0.91 & 0.21 & 0.64 & Residual del modelo \\
C2 & 1 & 3.4 & 0.78 & 0.37 & Residual del modelo \\
C3 & 1 & 14.76 & 3.38 & 0.06 & Residual del modelo \\
C4 & 1 & 0.16 & 0.04 & 0.84 & Residual del modelo \\
C5 & 1 & 0.12 & 0.03 & 0.87 & Residual del modelo \\
C6 & 1 & 0.61 & 0.14 & 0.7 & Residual del modelo \\
C7 & 1 & 2.8 & 0.64 & 0.42 & Residual del modelo \\
C8 & 1 & 2.17 & 0.5 & 0.48 & Residual del modelo \\
C9 & 1 & 8.06 & 1.84 & 0.17 & Residual del modelo \\
C10 & 1 & 7.65 & 1.75 & 0.18 & Residual del modelo \\
C11 & 1 & 2.15 & 0.49 & 0.48 & Residual del modelo \\
\hline
\end{tabular}




\begin{tabular}{cccccc}
\hline $\begin{array}{c}\text { CulxDenxFer } \\
\text { o AxBxC }\end{array}$ & $\begin{array}{c}\text { Grados de } \\
\text { libertad }\end{array}$ & $\begin{array}{c}\text { Suma de } \\
\text { cuadrados }\end{array}$ & $\begin{array}{c}\text { Valor de } \\
\text { F }\end{array}$ & $\begin{array}{c}\text { Valor de } \\
p\end{array}$ & $\begin{array}{c}\text { Error para la } \\
\text { interacción }\end{array}$ \\
\hline C12 & 1 & 0.33 & 0.08 & 0.78 & Residual del modelo \\
C13 & 1 & 0.71 & 0.16 & 0.68 & Residual del modelo \\
C14 & 1 & 1.19 & 0.27 & 0.6 & Residual del modelo \\
C15 & 1 & 0.57 & 0.13 & 0.71 & Residual del modelo \\
C16 & 1 & 1.31 & 0.3 & 0.58 & Residual del modelo \\
C17 & 1 & 0.001 & 0 & 0.98 & Residual del modelo \\
C18 & 1 & 8.12 & 1.86 & 0.17 & Residual del modelo \\
C19 & 1 & 3.65 & 0.84 & 0.36 & Residual del modelo \\
C20 & 1 & 0.81 & 0.18 & 0.66 & Residual del modelo \\
Total & 20 & 59.48 & 0.68 & 0.84 & Residual del modelo \\
\hline
\end{tabular}

\section{Conclusiones}

Los tres paquetes estadísticos generan información similar para la serie de experimentos en bloques completos al azar en arreglo de parcelas subdivididas (SE en DBCA en PS) en versiones libres o estudiantiles ( $\sin$ costo). La licencia anual para una PC para SAS es de USD \$2 000.00 y para InfoStat o InfoGen es de sólo USD \$50.00, los dos últimos son más amigables que SAS, al requerir de menos información para obtener los análisis estadísticos de interés.

En los tres softwares deben capturarse los coeficientes de los contrastes mutuamente ortogonales (CMO’s), su cálculo para las interacciones es más laborioso cuando aumenta el número de niveles dentro de cada factor. El usuario tiene la opción de construir sólo un subconjunto de CMO's o no ortogonales, que sean congruentes con los objetivos de la investigación. La significancia estadística de los valores de F de los CMO's podría considerarse como una guía práctica para diseñar otros análisis complementarios, como las comparaciones múltiples de medias de tratamientos o la aplicación de técnicas multivariadas.

Con la información que se presenta en este ensayo será más fácil extender su análisis de la SE en DBCA en PS para el caso de polinomios ortogonales (PO), superficies de respuesta, o a combinaciones de $\mathrm{PO}$ con CMO's.

\section{Literatura citada}

Balzarini, M. G.; González, L.; Tablada, M.; Casanoves, F.; Di Rienzo, J. A. y Robledo, C. W. 2008. Manual del usuario de InfoStat, Editorial Brujas, Córdoba, Argentina. 82-112 pp.

Balzarini, M. G. y Di Rienzo, J. A. 2016. InfoGen. FCA. Universidad Nacional de Córdoba, Argentina. http://www.info-gen.com.mx.

Di Rienzo, J. A.; Casanoves, F.; Balzarini, M. G.; González, L.; Tablada, M. y Robledo, C. W. 2008. InfoStat, versión 2008. Grupo InfoStat, FCA, Universidad Nacional de Córdoba. Argentina.

Gomez, K. A., Gomez, A. A. 1984. Statistical procedures for agricultural research. $2^{\text {nd }}$ (Ed.). John Wiley \& Sons, Inc. Printed in Singarore. 680 p. 
González, H. A.; Pérez, L. D. J.; Sahagún, C. J.; Norman, M. T. H.; Balbuena, M. A. y Gutiérrez, R. F. 2007. Análisis de una cruza dialélica completa de líneas endogámicas de maíz. Rev. Cienc. Agríc. Informa. 16(1):10-17.

González, H. A.; Sahagún, C. J. y Pérez, L. D. J. 2007. Estudio de ocho líneas de maíz en un experimento dialélico incompleto. Rev. Cienc. Agríc. Informa. 16(1):3-9.

Herrera, S. L. A. 2011. Análisis de la varianza de un grupo de experimentos en parcelas subdivididas. Revista de la Facultad de Ciencias Veterinarias, UCV. 52(1):59-72.

Juárez, M. J. A. y Corona, S. T. 1990. El análisis de experimentos por el método Papadakis. Rev. Chapingo. 71-72:110-113.

Martínez, G. A. 1988. Diseños experimentales. Métodos y elementos de teoría. Editorial Trillas. Primera Edición. México, DF. 756 p.

Matzinger, D. F.; Sprague, G. F. and Cockerham, C. C. 1959. Diallel Crosses of maize in experiments repeated over locations and years. Agron. J. 51(3):346-350.

Meneses, M.; Mejía I. C. J. A. y Villanueva, V. C. 2004. Cambios en los componentes de varianza genética al realizar selección combinada en una población de calabaza. Rev. Chapingo Ser. Hortic. 10(2):165-172.

Montgomery, D. C. 2010. Diseño y análisis de experimentos. Limusa-Noriega Editores. Segunda Edición, México, DF. 686 p.

Padilla, L. A.; González, H. A.; Pérez, L. D. J.; Rubí, A. M.; Gutiérrez, R. F.; Ramírez, D. J. F.; Franco, M. J. R. P. y Serrato, C. R. 2019. Programas para SAS e InfoStat para analizar una serie de experimentos en parcelas subdivididas. Universidad Autónoma del Estado de México. Primera Edición. Toluca, México. 45- 55 p.

Rebolledo, R. H. H. 2002. Manual SAS por computadora. Análisis estadístico de datos experimentales. Editorial Trillas. Primera edición. México, DF. 208 p.

Sahagún, C. J. 1990. Utilidad del análisis de varianza en el estudio de la interacción entre genotipos y ambientes. Xilonen. 1(1):21-32.

Sahagún, C. J. y Frey, K. J. 1990. Eficiencia de tres diseños experimentales para la evaluación de genotipos. Revista Chapingo. 71-72:114-122.

Sahagún, J. J. 1997. Estimación de varianzas genéticas con machos $\mathrm{S}_{0}$ y líneas hembras $\mathrm{S}_{1}$ en el Diseño II. Rev. Chapingo Ser. Hortic. 3(2):71-76.

Sahagún, C. J. 1998. Construcción y análisis de los modelos fijos, aleatorios y mixtos. Universidad Autónoma Chapingo (UACH). Departamento de Fitotecnia. Boletín técnico núm. 2. 65 p.

Sahagún, C. J.; Martínez, G. A. y Rodríguez, P. J. E. 2008. Problemas y métodos comunes del análisis de experimentos factoriales. Rev. Chapingo Ser. Hortic. 14(2):213-222.

SAS Institute Inc. 1989. SAS/STAT User's Guide, Version 6, Fourth Edition, Volume 1, Cary, NC, USA. 943 p.

Torres, F. J. L.; Mendoza, G. B.; Prassana, B. M.; Alvarado, G.; San Vicente, F. M. y Crossa, J. 2017. Grain yield and stability of white early maize hybrids in the highland valleys of Mexico. Crop Sci. 57(6):3002-3015.

Villa, M. A.; Herrera, L.; Díaz, I. y Sozzi, A. 2010. Análisis de varianza para diseños en parcelas subdivididas con tratamientos terciarios aleatorios y una factorial en las subparcelas. Ciencia. 18(2):126-136.

Walpole, R. E.; Myers, R. H. and Ye, K. 2012. Probability and Statistics for engineers and scientists. Prentice Hall-Pearson Education. Ninth Edition. USA. 791 p. 